\title{
The Benefits of MRI in the Prenatal Diagnosis of Congenital Diaphragmatic Hernia
}

Erick NESTIANUa, Cristina Guramba BRADEANU ${ }^{b}$, loana DRAGANc, Radu VLADAREANUa, d

a"Carol Davila" University of Medicine and Pharmacy, Bucharest, Romania

${ }^{b}$ Affidea Fundeni Imaging Center, Bucharest, Romania

'Columna Medical Center, Bucharest, Romania

${ }^{d}$ Obstetrics and Gynecology Department, Elias Emergency University Hospital, Bucharest, Romania

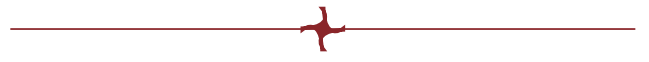

\begin{abstract}
Aims: We searched for correlations between ultrasound findings in pregnancies with congenital diaphragmatic hernia $(\mathrm{CDH})$ and magnetic resonance imaging (MRI) follow-up examinations; MRI was used to confirm and complete the investigation in these difficult cases. In some of them, new elements that ultrasound was not able to fully describe have been also brought. We were especially interested when MRI was superior to ultrasound.

Material and methods: This is a retrospective study of 12 pregnancies with congenital diaphragmatic hernia that were diagnosed in two major university clinics of Bucharest, Romania. Ultrasounds and MRI examinations were performed to evaluate pulmonary hypoplasia and correctly asses the herniated organs. We used standard international protocols and guidelines for calculating different parameters. All patients signed an informed consent before being enrolled in the study.

Results: We described the herniated organs, dimensions of the hernia and the remaining lung capacity, so that we could correctly evaluate the prognosis. We have also used the lung to head ratio (LHR) in an attempt to better determine the degree of lung hypoplasia.

Conclusions: High quality ultrasound followed by an MRI examination helped correctly assess the prognostic, treatment possibilities and total affected lung volume. It not only confirmed the diagnosis, but also offered new information that ultrasound was not able to provide.

Keywords: fetal diaphragmatic hernia, fetal abnormalities, prenatal diagnosis, ultrasound examination, fetal MRI.
\end{abstract}

\footnotetext{
Address for correspondence:

Nestianu Erick

Postal address: Costache Negri Str. 29, Bucharest 050552, Romania

Tel.: 0040722400261

Email: nestianuerick@gmail.com
}

Article received on the $30^{\text {th }}$ of August 2021 and accepted for publication on the $23^{\text {rd }}$ of September 2021 


\section{INTRODUCTION}

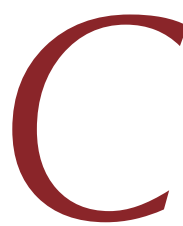

ongenital diaphragmatic hernia $(\mathrm{CDH})$ is a malformation with severe consequences in the development of the newborn, rising the morbidity and mortality during this period. Modern ultrasound techniques improved the diagnosis of this pathology, which offer an increased diagnostic accuracy when complemented by magnetic resonance imaging (MRI) examination (1). For the last 30 years, the intrapartum diagnosis of diaphragmatic hernia has been made using ultrasound (2). It is recognized as a syndrome that includes pulmonary hypoplasia, pulmonary immaturity, hypoplasia of the left heart and neonatal persistent pulmonary hypertension of the newborn (PPHN).

Due to the large difference in managing the newborn with hernia, differentiating the severity is of great help. Small defects may not require large interventions and can be more safely managed, while large defects usually need skilled intensive care (high frequency ventilation, $\mathrm{NO}$ administration and even ECMO).

New in utero treatments have appeared, such as fetal endoscopic (FETENDO) and fetoscopic endoluminal tracheal occlusion (FETO) treatments. The aim is to implant a balloon in the trachea and obstruct it, so that the lung can expand to the thoracic wall (3).

During the last 30 years, studies on lots of over ten million (4) pregnancies found an incidence of diaphragmatic hernia at approximately 2.3/10 000 (5), making it a rare condition. They also concluded that it represented about $8 \%$ of the total number of malformations that affect the fetus (6).

Most cases come from single pregnancies, and in about $40 \%$ of all cases, diaphragmatic hernia is also associated with other genetic syndromes (5), among which 21, 18 and 13 trisomy, but also $12 p$ tetrasomy, are more commonly encountered (7). Other anomalies include syndromes such as Fryns, Lange, Marfan, PallisterKillian and Donnai-Barrow $(8,9)$.

Age over 40 years, Caucasian race, alcohol consumption and smoking are considered risk factors. There is a 1:0.69 male to female ratio of the malformity (4). The mean detection age is 24.2 weeks (10). A higher incidence of premature birth, intra-uterine growth restriction and death (antepartum as well as postpartum) has been also noted, and mortality at one year of age is around $45.8 \%(11)$.

Usually, the lung on the affected side is hypoplastic, while the lung on the contralateral side is only partially affected (12).

\section{MATERIAL AND METHODS}

The article is based on a retrospective study conducted between mid-2019 and mid-2021. We studied all new information that MRI examination could reveal about pregnancies after ultrasound failure to provide clear data. We only took an interest in the morphological elements observed.

Patients were selected from those who underwent a second trimester fetal morphology screening in two university hospitals. It is important to note that these hospitals attract a large number of patients from a wide area, and as such, examined cases included a number of patients who presented for a second opinion investigation as well as other complicated cases that lesser clinics were unable to clarify.

The inclusion criteria were as follows: pregnant women who underwent first trimester fetal morphology screening, and had a negative test for aneuploidies; $\mathrm{CDH}$ was the only identified malformation; patients accepted to undergo an MRI examination; pregnancy was spontaneous or after by assisted human reproduction (AHR).

We obtained approval from the Ethical Committee of the examination centers prior to conducting this study. All patients have also signed an informed consent prior to inclusion.

Patients were rigorously monitored throughout the pregnancy, as per the International Society of Ultrasound in Obstetrics and Gynecology (ISUOG) protocols, with follow up ultrasound in both the second and third trimesters, complying with all guideline parameters (13).

Ultrasound examination was performed on high-end devices, with a dedicated software for obstetrics examination. They used mode B ecodoppler and are equipped with a convex transducer with a wide band and frequency between 2 and $8 \mathrm{Mhz}$. We adapted the power as needed, generally using a lower setting, so that we could obtain a good image depth and special resolution in order to better describe the existing lesions. 
After the original ultrasound diagnosis was made, patients were informed about the implications and possible complications of the disease and were advised to undertake a follow-up MRI examination. We searched for direct (identifying an abdominal viscera in the thorax) or indirect (mediastinal and heart axis shifting) signs of herniation found on initial exam. The optimal gestational age for the MRI examination was between 31 and 32 weeks.

We used a 1.5 Tesla MRI machine, with no gadolinium contrast agents, as suggested by the current international recommendations. Body coils were used to enhance the image. The used sequences include Fast Imaging Employing Steady State Acquisition (FIESTA, FOW de 450/500 mm, TR of 5,2 ms, TE of 2,4 ms), Single Shot Fast Spin Echo (SSFSE, FOW de 450/500 mm, TR of $534.4 \mathrm{~ms}$, TE of $160.2 \mathrm{~ms}$ ), Diffusion Weighted Image (DWI, FOW de 450/500 mm, TR of $6.2 \mathrm{ms,}$ TE of $3.1 \mathrm{~ms}$ ) and Liver Acquisition with Volume Acceleration (LAVA, FOW de 450/500 mm, TR of $6.2 \mathrm{~ms}$, TE of $3.1 \mathrm{~ms}$ ). Slice thickness was between 4 and $6 \mathrm{~mm}$ (14).

During the investigation, the mother was in the supine or lateral position, without sedation, as per ISOUG recommendations (15).

Prognosis was evaluated using the LHR (lung to head ratio) corelated with the gestational age following the estimations made by Jani etal $(5,16)$.

\section{RESULTS}

total of 9879 patients underwent ultrasound screening. We identified a total of 324 cases with malformations, of which 12 presented with $\mathrm{CDH}$ and were therefore recommended to have a MRI examination.

Ultrasound biometry was in concordance with the MRI biometry in all examined cases. UItrasound diagnosis is made only after objectifying both direct signs of diaphragmatic hernia, including stomach, intestines or liver that have ascended in the thoracic cavity, and indirect signs, including mediastinal shifting on the contralateral side, and cardiac axis shifting (11).

MRI differentiates lung from liver and other herniated structures from the mediastinum better than ultrasound, and it also differentiated small bowel loops from the colon. Early examinations identify what organs have ascended through the defect and search for other malfor- mations. Later examinations evaluate the pulmonary volume and pulmonary hypoplasia (12).

Some authors suggest the use of a lung and head circumference ratio (LHR). In our study we used the technique proposed by Jani et al $(5,16)$.

Patients who underwent an MRI scan had a gestational age between 21.3-34.2 weeks at the time of the examination. We were most interested in the size of the defect, what organs herniated in the thorax and their impact on the lungs; we took all this into consideration when we decided the prognosis and the treatment procedures. Ultrasound always correctly identified the defect location (left or right herniation).

We had a single twin pregnancy, while the rest were single pregnancies.

The defect was on the left side in 10 of the 12 examined cases $(83.3 \%)$.

In all patients, at least three abdominal structures were herniated in the thorax. Most commonly we found the presence of intestinal loops (all 12 patients), followed by segments of the co-

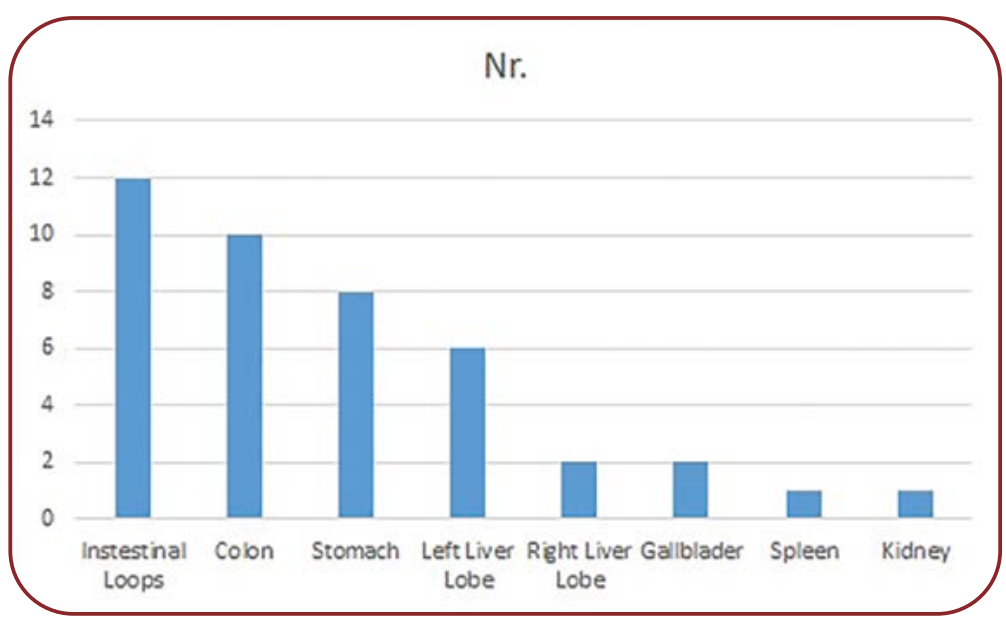

FIGURE 1. Herniated organs found during the MRI study

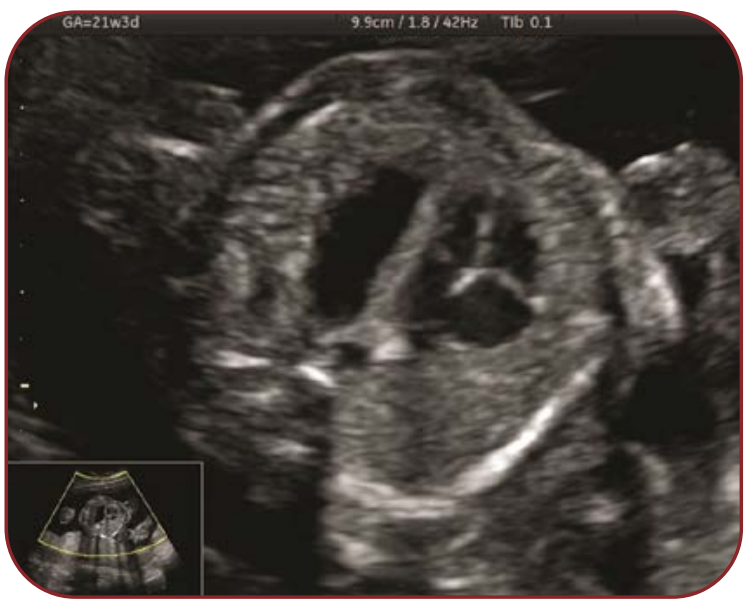

FIGURE 2. Echography transverse section of the thorax revealing the four-chamber view of the fetal heart 
Ion (10 out of 12 cases), and stomach (8 of 12 cases) (Figures 1 and 2).

Herniation of the liver was present in eight $(66.7 \%)$ patients, the left liver lobe herniated in six (50\%) patients, and the right liver lobe in only two $(16.7 \%)$ subjects (Figures 3, 4 and 5).

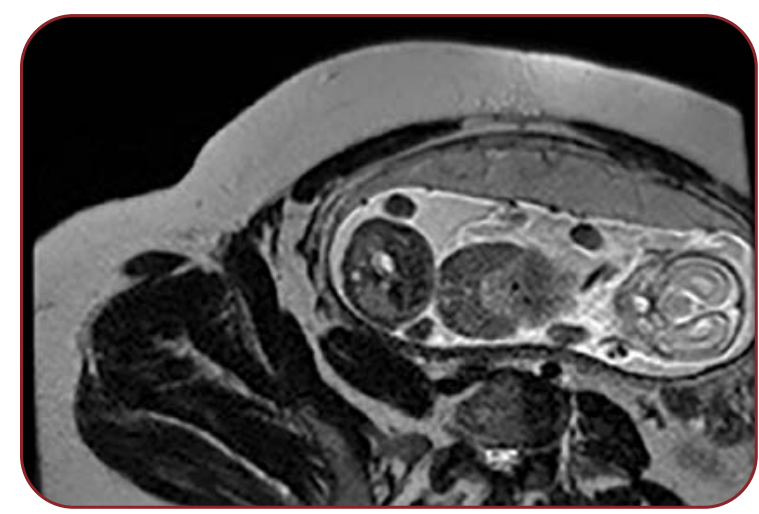

FIGURE 3. MRI SSFSE aquisition T2 weighted axial section of a twin pregnancy

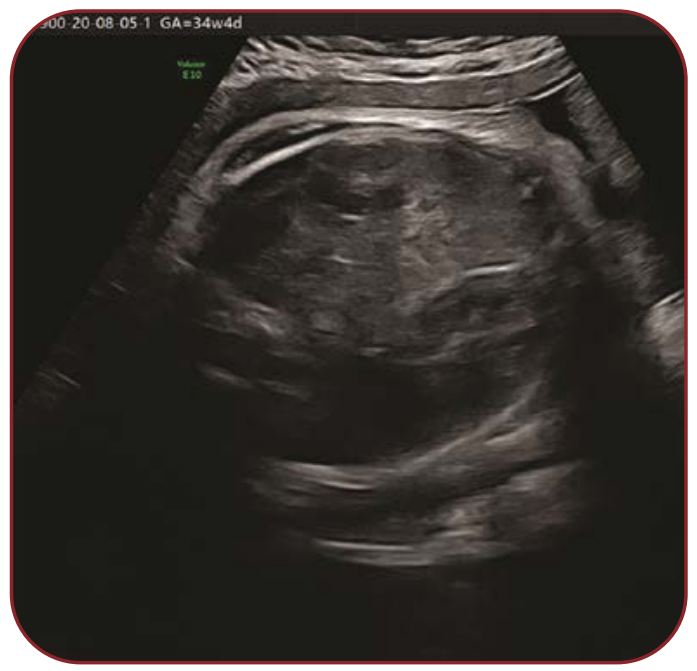

FIGURE 4. Echography transverse section of the thorax; the heart is displaced to the left hemithorax

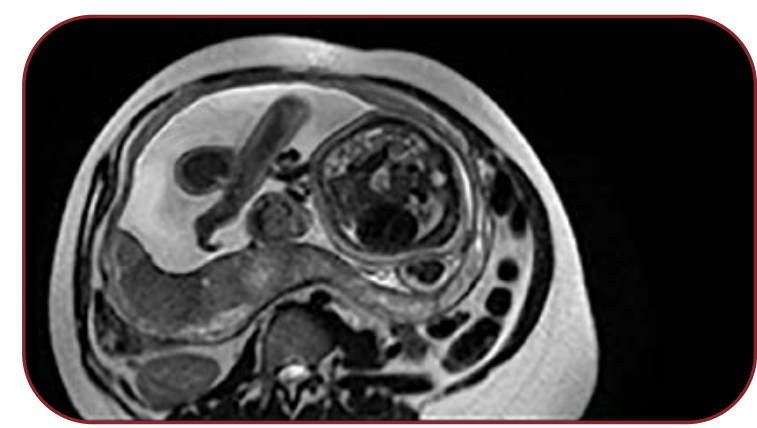

FIGURE 5. MRI SSFSE aquisition T2 weighted axial image, clearly showing the herniation of the right liver lobe and the gallblader

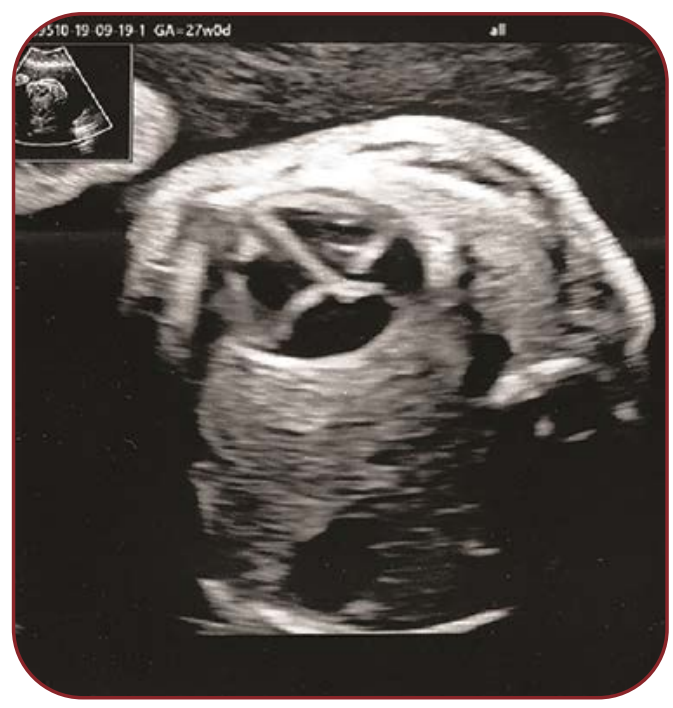

FIGURE 6. Echography transverse section of the thorax revealing the four-chamber view of the fetal heart, in which a left diaphragmatic hernia is observed

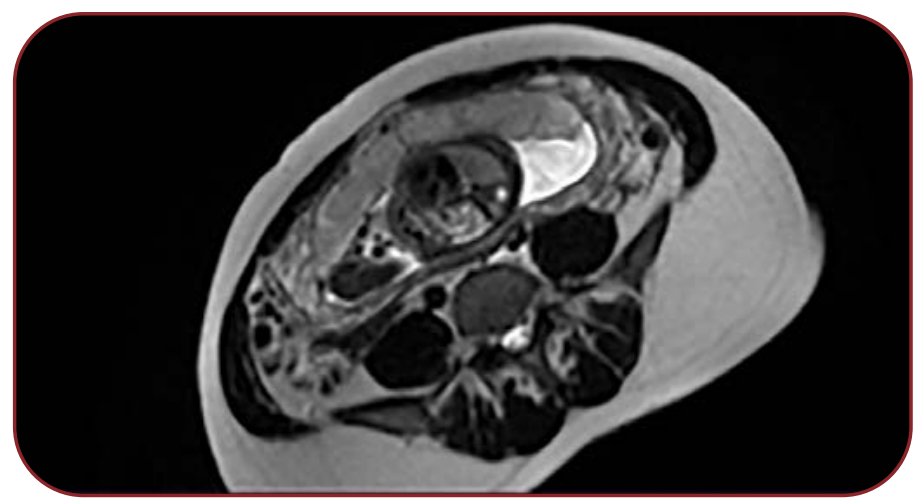

FIGURE 7. MRI SSFSE aquisition T2 weighted axial image, in which the stomach, small bowel loops, and a part of the colon can be seen

Intestinal loops were seen in the thorax in eight $(66.7 \%)$ cases, of which four involved the left liver lobe and two, the right liver lobe (Figure 6). Only the MRI examination helped differentiate between the intestinal loops and the colonic segments (Figure 7).

The MRI scan differentiated the lung from the liver three times, when the ultrasound examination was not able to do that (Figures 7, 8 and 9). Two of the three identified were those that presented the right sided herniation, in which the right lung was collapsed and pushed to the left by the liver. The ultrasound scan could not determine if the lung had shrunk in volume, but the MRI investigation provided the answers and confirmed a reduction in total lung volume ( $\mathrm{Fi}$ gures 10,11 and 12). In the last case, it differen- 


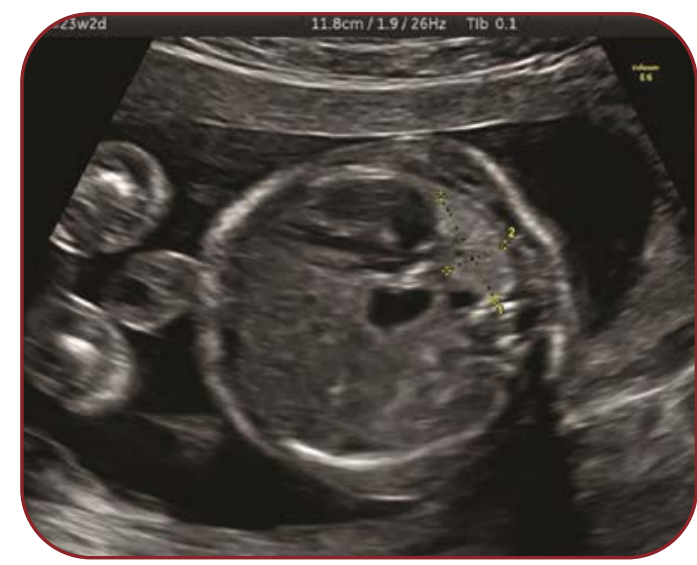

FIGURE 8. Echography transverse section of the thorax that reveals a hipoplasic right lung, a partialy collapsed left lung and the heart that is placed in the right hemithorax

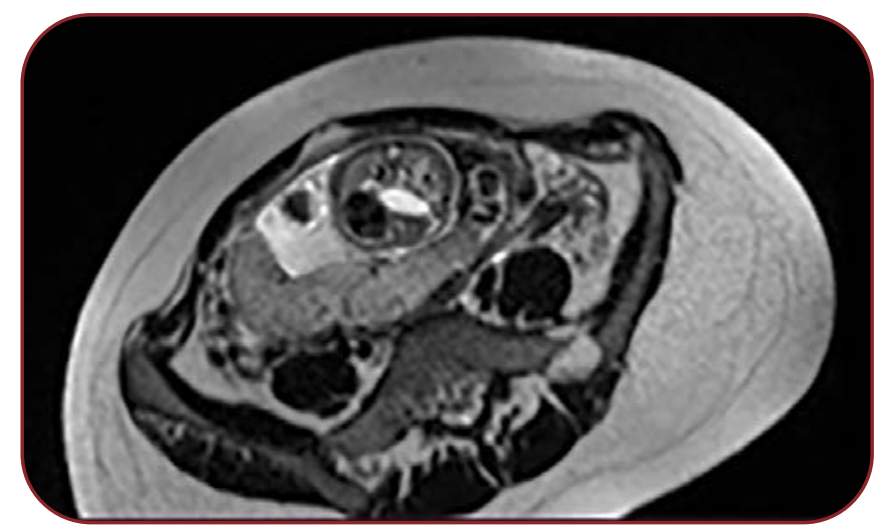

FIGURE 9. MRI SSFSE aquisition T2 weighted axial image

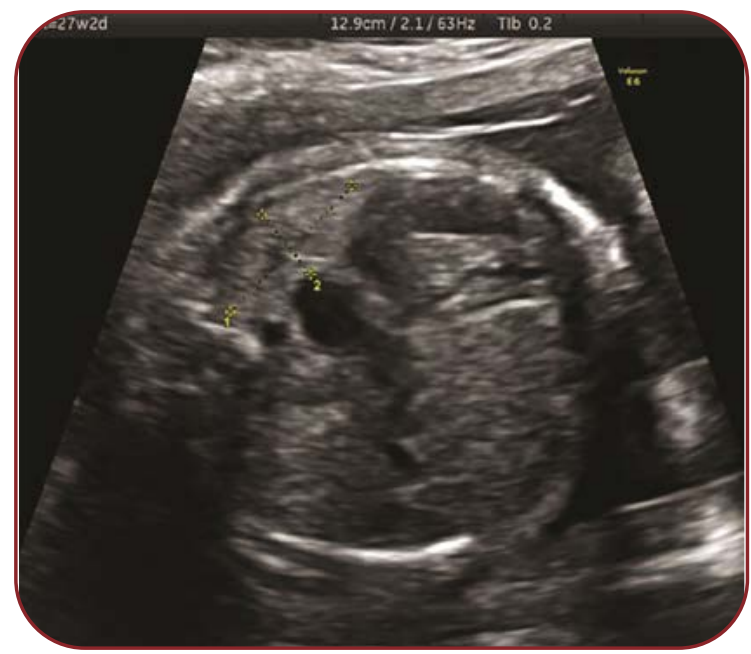

FIGURE 10. Echography left diaphragmatic hernia

tiated the lung and liver of a left sided defect, in which the liver ascended in the thorax It also showed the herniation of the left kidney (Figures 13 and 14).

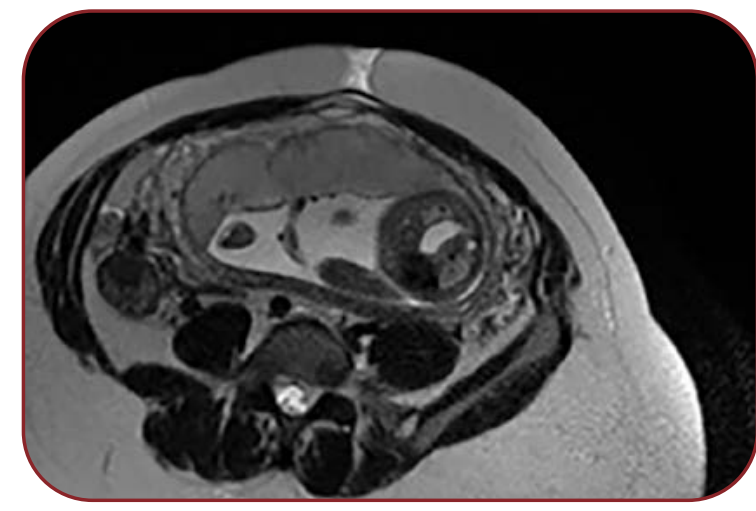

FIGURE 11. MRI SSFSE aquisition T2 weighted axial image

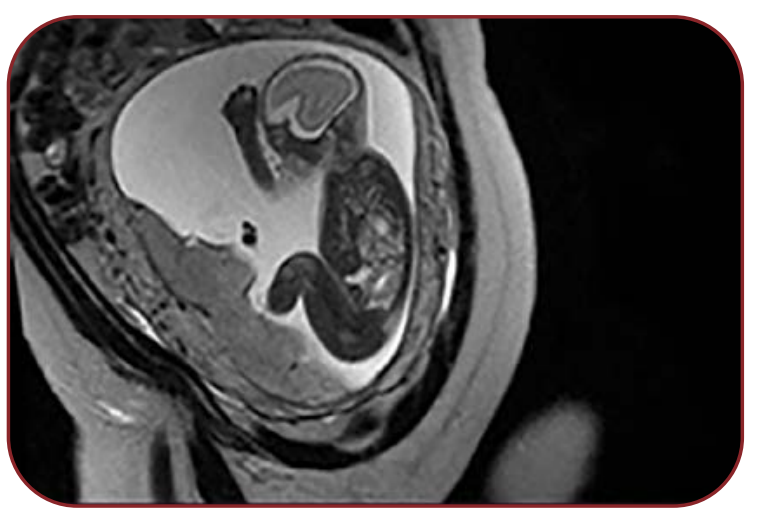

FIGURE 12. MRI SSFSE aquisition T2 weighted sagital image of the same case as that shown in Figure 13

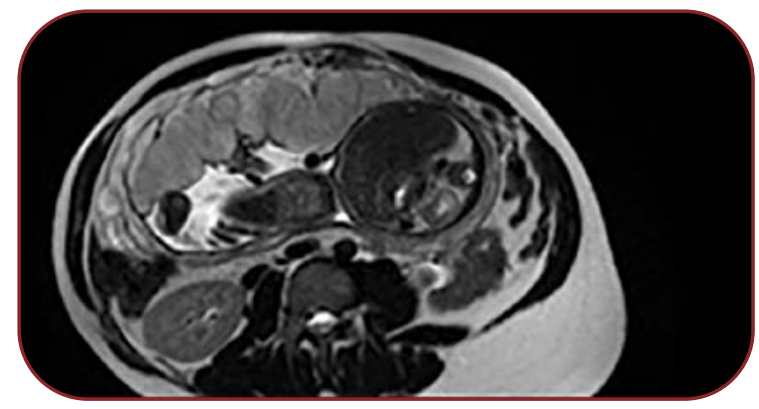

FIG URE 13. MRI SSFSE aquisition T2 weighted axial image that sows the herniation of the left kidney

When we calculated the LHR, we found four situations in which it was 1.5 (good prognosis with $72 \%$ survival rate), four cases where it was 1.2 (a good prognosis, but with only $68 \%$ survival rate), and the last four cases had an LHR of 0.9 and 1.0, respectively (bad prognosis, with only about $32 \%$ survival rate).

The ratio between the calculated LHR and the estimated one adapted to the gestational age 


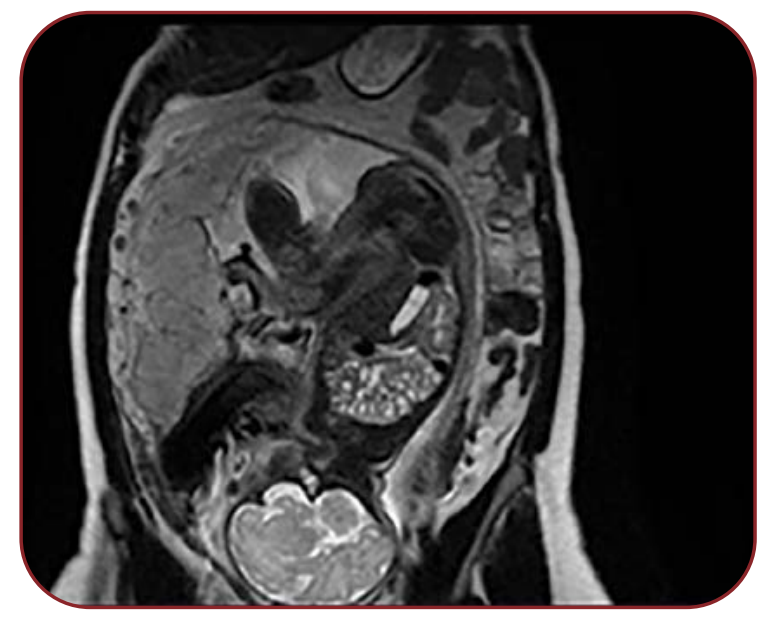

FIGURE 14. MRI SSFSE aquisition T2 weighted sagital image of the same case as that shown in Figure 9

ranged between $45 \%$ and $62 \%$, according to the defect size and severity.

All mothers were directed to specialized medical centers at the due date, and all surviving infants needed specialty neonatal ICU treatments as well as pediatric and pediatric surgery evaluation.

\section{DISCUSSIONS}

P atients studied by us do not form a numerous lot, but considering the low incidence of these malformation, the incidence is actually a little bit higher. This is probably because university hospitals gather many difficult cases in a wide region, that other medical centers cannot handle, and also a big number of patients who look for a second opinion. Our incidence was higher by a substantial margin compared to the literature (12.1 vs $2.3 / 10$ 000) (5). This is probably explained by the fact that university hospitals gather patients from a wide area, and cumulate a big number of rare pathologies, thus the real incidence of this malformity is probably lower than what we discovered in this study.

Findings were consistent with the literature, and as such, the majority of cases (10 out of 12) presented with a left side herniation. The most common herniated structures were the small bowel, colon, stomach and liver (17). Eight cases presented herniation of the liver - six involving the left liver lobe and two the right liver lobe along with the gall bladder. A single patient had a kidney displaced through the herniation defect.
We calculated the LRH in all cases in order to better assess the level of lung hypoplasia, from which we then estimated the survival rate. $\mathrm{Na}$ turally, better results were observed when the defect was smaller and only the small bowel, stomach and a little bit of the liver were discovered in the thoracic cavity, as opposed to cases when a larger part of the liver and even a kidney were seen to migrate through the defect. We did not calculate the lung volume, as it is not the subject of this article.

In our study, the MRI examination has provided some extra information when comparing it to ultrasound as follows: in a few cases, it could correctly differentiate between the collapsed lung and the liver lobe that ascended in the thorax, thus allowing a much better evaluation of the LHR and the remaining pulmonary volume; in one situation, it revealed that a kidney also ascended in the thoracic cavity through the diaphragmatic defect, even though this was the case; in 10 cases, it distinguished between the small bowel and segments of the colon that herniated through the defect; in two cases, it confirmed the herniation of the right liver lobe, along with the gall bladder; ultrasound was not able to correctly identify the gall bladder and small bowel that herniated; it was able to regularly and correctly differentiate between small intestinal loops and colon segments (9).

Most pregnancies were unique, and only a single case of twin pregnancy was observed.

Calculating the LHR and the ratio between the calculated LHR and the estimated value corresponding to the age of the pregnancy proved useful in determining the prognosis and degree of pulmonary hypoplasia; it also corelated with the herniation size; and a larger amount of herniated organs in the thorax determined a bigger degree of pulmonary hypoplasia (18).

We believe that the discovery rate of such pathologies will increase with expansion of specialized medical centers. The correlation between findings provided by ultrasound investigation and those offered by MRI examination will improve the final outcome of patients. The family must be counseled to complete the necessary investigations and to approach specialized healthcare, so that we can offer the best chances of survival to the newborn. The lack of developed medical centers will mean that some cases 
will be missed, which can lead to a more complex and difficult postpartum treatment.

Finally, we believe that if the delivery is not made in a specialized medical center that does not have a specialized ICU and infantile surgery ward, the newborn might not be able to get the needed medical attention in time.

\section{CONCLUSIONS}

The he MRI examination was successful in differentiating collapsed lung tissue form herniated liver tissue. Also, it has proved useful in proving herniation of the digestive system from the abdominal cavity with the further benefit of also identifying what parts they belong to (small bowel, cecum, etc). It also allowed a more precise calculation of the degree of pulmonary affliction and pulmonary volume than the ultra- sound examination, so that a more correct approach can be decided for each individual patient.

In some cases, it can even show herniation of some structure that can be originally missed on the classical ultrasound examination, like kidneys.

Magnetic resonance imaging can also be used to confirm that the biometric measurements from the ultrasound examination were correctly measured.

In the current state, ultrasound remains the go to imagistic investigation for fetal malformations, while MRI investigation comes only as an auxiliary method to help us better evaluate the pathology and asses the prognosis.

Conflicts of interest: none declared. Financial support: none declared.

\section{R}

1. Fernández-Perea $Y$, Lutgardo G-D, Javier S, et al. Ultrasound, Echocardiography, MRI, and Genetic Analysis of a Fetus with Congenital Diaphragmatic Hernia and Partial 11q Trisomy. Case Rep Obstet Gynecol 2017:1-4.

2. Jayalaxmi SA, Aruna G, Deepak J et al. Hernia in Neonates: Our Institutional Experience. J Indian Assoc Pediatr Surg 2018;23:131-139.

3. Deprest J. Gratacos E, Nicolaides KH. Fetoscopic tracheal occlusion (FETO) for severe congenital diaphragmatic hernia: evolution of a technique and preliminary results. Ultrasound Obstet Gynecol 2004;24:121-126.

4. McGivern Mark R, Best Kate E, Rankin J, et al. Epidemiology of congenital diaphragmatic hernia in Europe: a register-based study. Arch Dis Child Fetal Neonatal Ed 2015;100:F137-F144.

5. Mieke C, Jacques J. Diagnosis of Congenital Diaphragmatic Hernia. In: [book author] D. Prayer. Fetal MRI. Berlin-Haidelberg: Springer-Verlag, 2011, pp. 229-339.

6. Crombleholme TM, D'Alton ME, Malone FD, Bianchi DW.
Fetology Diagnostic and Management of the Fetal Patient. Second edition.

New York: Mc Graw Hill, 2010, p 37.

7. The Fetal Medicine Foundation. https://fetalmedicine.org/education/ fetal-abnormalities/thorax/ diaphragmatic-hernia. fetalmedicine.org. [Interactiv] [Last Cited: 19 Jan 2021.] https://fetalmedicine.org/education/ fetal-abnormalities/thorax/ diaphragmatic-hernia.

8. Witters I, Legius E, Moerman P, et al. Associated malformations and chromosomal anomalies in 42 cases of prenatally diagnosed diaphragmatic hernia.

Am J Med Genet 2001;103:278-282.

9. Tovar, Juan A. Congenital Diaphragmatic Hernia.

Orphanet J Rare Dis 2012;7:1.

10. Clementi M, Garne E, Haeusler M, et al. Congenital diaphragmatic hernia: evaluation of prenatal diagnosis in 20 European regions. Ultrasound Obstet Gynecol 2002;19:323-333.

11. Beth M, Kline-Fath. Congenital Diaphragmatic Hernia. In: [book author] Dorothy I. Bulas, Ray Bahado-Singh Beth M. Kline-Fath. Fundamental and Advanced Fetal Imaging Ultrasound and MRI. se: Wolters Kluwer Health 2015, 17, pp 583-595.

12. Moya F R, Thomas V L, Romaguera J, et al. Fetal lung maturation in congenital diaphragmatic hernia. Am J Obstet Gynecol 1995;173:1401-1405.

13. ISUOG. Performance of the routine mid-trimester fetal ultrasound scan. ISUOG. org. [Interactiv] 2011;1:116-126.

14. Taylor G, Atalabi OM, Estroff J. Imaging of congenital diaphragmatic hernias. Pediatric Radiology 2008;39:1-16.

15. Prayer D, Malinger G, Brugger PC, et al. ISUOG Practice Guidelines: performance of fetal magnetic resonance imaging. Ultrasound Obstet Gynecol 2017;49:671-680.

16. UCSF University of California, San Francisco.

https://fetus.ucsf.edu/cdh. [Interactiv] Benioff Children*s Hospitals. [Citat: 25 Jan 2021.]

17. Pilu G, Nicolaides KH. Diagnosis of fetal abnormalities - The 18-23-week scan. New York, London: The Parthenon Publishing Group, 1999. ISBN 1-85070-492-9.

18. Salomon LJ, Alfirevic Z, Berghella V, et al. Practice guidelines for performance of the routine mid-trimester fetal ultrasound scan. Ultrasound Obstet Gynecol 2011;37:116-126. 